\title{
Effects of temperature on domain-growth kinetics of fourfold-degenerate $(2 \times 1)$ ordering in Ising models
}

\author{
Høst-Madsen, Anders; Shah, Peter Jivan; Hansen, Torben; Mouritsen, Ole G.
}

Published in:

Physical Review B

Link to article, DOI:

10.1103/PhysRevB.36.2333

Publication date:

1987

Document Version

Publisher's PDF, also known as Version of record

Link back to DTU Orbit

Citation (APA):

Høst-Madsen, A., Shah, P. J., Hansen, T., \& Mouritsen, O. G. (1987). Effects of temperature on domain-growth kinetics of fourfold-degenerate $(2 \times 1)$ ordering in Ising models. Physical Review B, 36(4), 2333-2336.

https://doi.org/10.1103/PhysRevB.36.2333

\section{General rights}

Copyright and moral rights for the publications made accessible in the public portal are retained by the authors and/or other copyright owners and it is a condition of accessing publications that users recognise and abide by the legal requirements associated with these rights.

- Users may download and print one copy of any publication from the public portal for the purpose of private study or research.

- You may not further distribute the material or use it for any profit-making activity or commercial gain

- You may freely distribute the URL identifying the publication in the public portal 


\title{
Effects of temperature on domain-growth kinetics of fourfold-degenerate $(2 \times 1)$ ordering in Ising models
}

\author{
Anders Høst-Madsen, Peter Jivan Shah, Torben Vendt Hansen, and Ole G. Mouritsen* \\ Department of Structural Properties of Materials, The Technical University of Denmark, \\ Building 307, DK-2800 Lyngby, Denmark
}

(Received 19 March 1987)

\begin{abstract}
Computer-simulation techniques are used to study the domain-growth kinetics of $(2 \times 1)$ ordering in a two-dimensional Ising model with nonconserved order parameter and with variable ratio $\alpha$ of next-nearest- and nearest-neighbor interactions. At zero temperature, persistent growth characterized by the classical growth exponent $n=\frac{1}{2}$ is found for $\alpha \geq 1$, whereas the domain boundaries become pinned and the growth stops for $\alpha<1$. For finite temperatures and $\alpha \geq 1$, the growth exponent is found to be temperature independent in a wide regime, and for $\alpha \leq 1$ the domain walls unpin and growth resumes.
\end{abstract}

Two-dimensional Ising models have served as the principal class of microscopic interaction models in the study of domain-growth kinetics and the dynamics of random interfaces. $^{1-17}$ They have had seminal importance in establishing the validity of dynamical scaling and in clarifying aspects of a universal classification of growth processes. The main reasons for the usefulness of the Ising models are their relative simplicity and their capacity of possessing a rich phase structure including commensurate and incommensurate modulated phases with a variety of symmetries. Furthermore, the isomorphic relationship of Ising models to lattice-gas models has made them applicable to various surface-adsorption problems. ${ }^{6,12}$

In this Brief Report we report on new computersimulation results for the domain-growth kinetics of a two-dimensional Ising model with $(2 \times 1)$-ordered phases on a square lattice. The kinetics is studied for a nonconserved order parameter as a function of temperature and ratio $\alpha$ of next-nearest-neighbor (NNN) and nearestneighbor (NN) coupling constants. The ground-state symmetry of the model is the same as that of atomic oxygen chemisorbed on (110) surfaces of tungsten. ${ }^{6,8,18}$ Our results supplement the recent and very extensive study by Sadiq and Binder ${ }^{8}$ of dynamical scaling and growth in the same model for conserved as well as nonconserved magnetization.

The model Hamiltonian, on which we have based our calculations,

$H=-J_{\mathrm{NN}} \sum_{\langle i, j\rangle}^{\mathrm{NN}} \sigma_{i} \sigma_{j}-J_{\mathrm{NNN}} \sum_{\langle i, j\rangle}^{\mathrm{NNN}} \sigma_{i} \sigma_{j}, \sigma_{i}= \pm 1$,

allows for a variable ratio, $\alpha \equiv J_{\mathrm{NNN}} / J_{\mathrm{NN}}$, of strengths of the isotropic next-nearest- and nearest-neighbor interactions. For $J_{\mathrm{NN}}<0$, the ground state is simple $(1 \times 1)$ antiferromagnetic for $\alpha<\frac{1}{2}$ and $(2 \times 1)$ superantiferromagnetic for $\alpha>\frac{1}{2}$. At $\alpha=\frac{1}{2}$, the two types of ordering are degenerate. ${ }^{19}$ The order-parameter degeneracy in the three regimes is described by $n=1\left(\alpha<\frac{1}{2}\right), n=3$ $\left(\alpha=\frac{1}{2}\right)$, and $n=2\left(\alpha>\frac{1}{2}\right)$. The corresponding number of thermodynamically degenerate ordered domains at low temperature is then $p=2 n$. Sadiq and Binder's study ${ }^{8}$ in the case of nonconserved magnetization was carried out at $\alpha=1$ for a few selected temperatures below the critical point $T_{c}$. In this paper, we report results for a series of ratios on both sides of $\alpha=1$. For a few values of $\alpha$, a systematic study as a function of temperature is undertaken. It turns out that the low-temperature kinetics is very sensitive to the value of $\alpha$. The kinetics of the model is, in our study, governed by a single-site spin-flip mechanism subject to the Metropolis criterion, ${ }^{20}$ combined with a random visitation of lattice sites. Thus the order parameter is a nonconserved quantity.

The kinetics of the spin ordering following global thermal quenches of the model from $T_{i} \sim \infty$ to $T_{f}<T_{c}(\alpha)$ is studied by Monte Carlo simulation of the spin dynamics, using finite lattices subject to periodic boundary conditions. ${ }^{21}$ Different lattice sizes have been used in order to assess finite-size effects. The bulk of the results reported below refers to lattices with $N=100^{2}$ and $200^{2}$ spins. The time evolution has been followed up to $t=500$, where the time is measured in units of Monte Carlo steps per site (MCS/S). Ensemble averages are obtained by averaging over a large number of independent quenches. A number of quantities can be used to monitor the growth of the ordered domains as a function of time. We shall here use (a) the length scale ${ }^{8}$

$$
L(t)=\sqrt{N}\left[\psi_{1}^{2}(t)+\psi_{2}^{2}(t)\right]^{1 / 2},
$$

derived from the two components of the time-dependent order parameter, (b) the excess internal energy $\Delta E(t)=E(t)-E\left(T_{f}\right)$ measuring the nonequilibrium energy stored in the entire domain-boundary network, where $E\left(T_{f}\right)$ is the equilibrium energy at $T_{f}$, and (c) the average linear domain size $R(t)$ derived from the complete domain distribution function. This distribution function is determined for individual spin configurations by taking full account of the four different types of domains and the six different domain walls ${ }^{8}$ which may arise. We have chosen to ascribe a given spin to a specific domain type if this spin, and at least three of its nearest neighbors, produces a spin pattern compatible with the ordering of that domain type. 
In Fig. 1 are shown the results for the various measures of the zero-temperature growth as a function of time in the case $\alpha=1$. It is found that the data for $t \gtrsim 10$ are accurately represented by the growth laws

$$
R(t) \sim L(t) \sim \Delta E^{-1}(t) \sim t^{n},
$$

with a common exponent value of $n \simeq 0.50$. For $t \gtrsim 500$ in the $100 \times 100$ lattice and for $t \gtrsim 900$ in the $200 \times 200$ lattice, finite-size effects due to "slab formation" of a percolating cluster ${ }^{3}$ are found to set in. Sadiq and Binder ${ }^{8}$ found the same growth laws, ${ }^{22} \mathrm{Eq}$. (3), to hold for $T=0.36 T_{c}$ and $0.63 T_{c}$, but did not notice that the power laws hold even at $T=0$. Thus the growth is described by the classical Lifshitz-Allen-Cahn growth law. ${ }^{23}$ The finding of a common growth exponent for length scales and inverse excess energy supports the concept of dynamical scaling. ${ }^{8}$ That the model indeed grows at zero temperature is a highly nontrivial finding. Other models with discrete single-site variables on square lattices are found to freeze into metastable glassy structures at low temperatures. ${ }^{14,24-27}$ In fact, the same model as studied here but with conserved magnetization also freezes into a glasslike domain structure at $T=0 .{ }^{8}$

Figure 2, using data for $\Delta E(t)$, shows that the growth behavior at $T=0$ is strongly dependent on the energetics of the model, that is the value of $\alpha$. The data for $R(t)$ and $L(t)$ show the same behavior. The results indicate that, as far as the zero-temperature kinetics is concerned, the point $\alpha=1$ is a very special one. For $\alpha \geq 1$, the domains

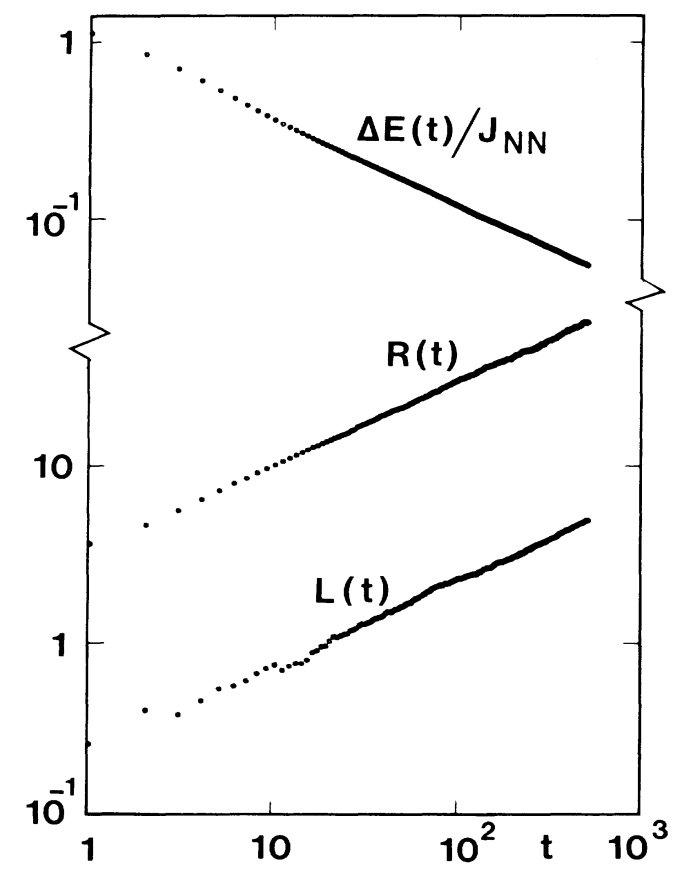

FIG. 1. Log-log plot vs time of length scales $L(t)$ and $R(t)$ and excess energy $\Delta E(t)$ for a quench to $T_{f}=0$ in the case $\alpha=1$. The results are obtained from Monte Carlo calculations on a system with $200^{2}$ spins. The time $t$ is in units of MCS/S. The thin solid lines denote the asymptotic power laws. Eq. (3), with the exponent value $n \simeq \frac{1}{2}$. grow persistently and the kinetics is independent of the value of $\alpha$ and universally described by the classical Lifshitz-Allen-Cahn exponent $n=\frac{1}{2}$. In contrast, for $\alpha<1$ the domain growth ceases after some time $t_{0}$, the value of $t_{0}$ being smaller the smaller the value of $\alpha$. It should be noted that the point of degeneracy, $\alpha=\frac{1}{2}$, is somewhat pathological since $T_{c}\left(\alpha=\frac{1}{2}\right) \simeq 0 .{ }^{19}$

As Fig. 2 indicates, the ceasing of growth and the underlying domain-wall pinning become effective in a very abrupt manner as $\alpha$ is lowered below $\alpha=1$. This conspicuous effect may be understood by considering the excess domain-wall energies, $\delta E$, per wall spin at $T=0$ for the six different types of domain walls. ${ }^{8}$ (See inset in Fig. 4.) At late times, those walls with the lowest $\delta E$ will control the growth. The superheavy $(\mathrm{SH})$ and the superlight (SL) walls have $\delta E_{\mathrm{SH}}=\delta E_{\mathrm{SL}}=(1+2 \alpha) J_{\mathrm{NN}}$; the heavy $(\mathrm{H})$, the light (L), and the $45^{\circ}$ walls have $\delta E_{\mathrm{H}}=\delta E_{\mathrm{L}}=\delta E_{45}=\alpha J_{\mathrm{NN}}$; and the antiphase (A) walls have $\delta E_{\mathrm{A}}=(-1+2 \alpha) J_{\mathrm{NN}}$. The SH and SL walls are always very unfavorable independent of $\alpha$. For $\alpha>1$, the $\mathrm{H}, \mathrm{L}$, and $45^{\circ}$ walls are dominant, and for $\alpha<1$ the $\mathrm{A}$ walls dominate. At $\alpha=1$, the $\mathrm{H}, \mathrm{L}, 45^{\circ}$, and A walls are degenerate. The point is now, that whenever the $45^{\circ}$ walls are mixed with horizontal and vertical walls (Fig. 4), growth will proceed via kink formation and propagation of these kinks through vertices. In the absence of $45^{\circ}$ walls,

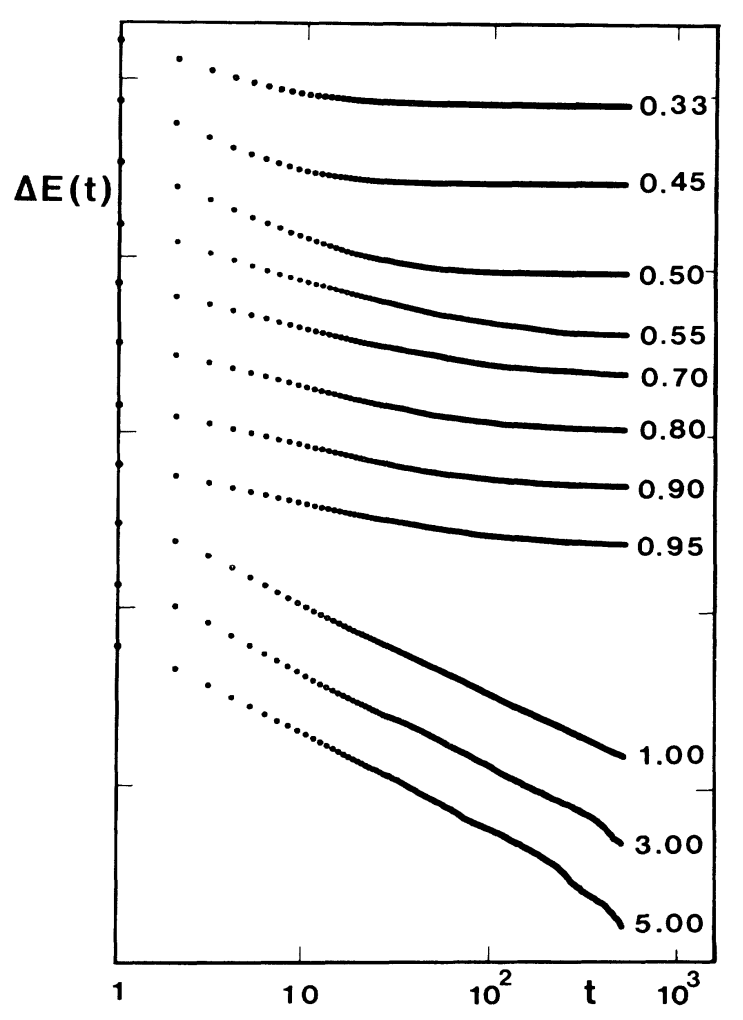

FIG. 2. Log-log plot of the excess energy $\Delta E(t)$ (in arbitrary units) vs time for quenches to $T_{f}=0$ in the case of varying $\alpha$. The results are derived from Monte Carlo calculations on lattices with $100^{2}$ spins. The time $t$ is in units of MCS/S. The curves are labeled by the value of $\alpha$. 
the kinks will be absorbed at the square vertices and the walls will be pinned. A similar behavior has been observed in Potts models on square lattices. ${ }^{24,27}$

The texture of the domain pattern very strongly reflects the growth characteristics given by the time evolution of the macroscopic properties, such as $R(t)$. For $\alpha \geq 1$, the domains of the four types of ordering grow in a regular and compact fashion (see, e.g., the microconfigurations in Ref. 8) until they have reached sizes comparable to the size of the entire system. Conversely, for $\alpha<1$ the domain growth stops and a pinned polycrystalline structure is formed before interference with the finite size of the lattice is observed. As $\alpha$ is lowered toward $\alpha=\frac{1}{2}$, the average domain size in the pinned state decreases and the texture of the domain pattern appears more and more glassy. At $\alpha=\frac{1}{2}$, a glassy frozen-in configuration of the six degenerate domains is obtained. The average domain size is very small. For $\alpha<\frac{1}{2}$, a stationary glassy polycrystalline domain structure of $(1 \times 1)$ antiferromagnetic domains is formed at early times.

The temperature dependence of the growth process at $\alpha=1$ is illustrated via data for $R(t)$ in Fig. 3. The data for $\Delta E(t)$ and $L(t)$ show a similar dependence, except that the log-log curves of these quantities are perfectly linear up to $T \simeq 0.95 T_{c}$, whereas the $R(t)$ curves deviate progressively from linearity for $T \gtrsim 0.7 T_{c}{ }^{28}$ For $T \lesssim 0.7 T_{c}$, the growth exponent is independent of temperature and assumes the same value, $n \simeq 0.50$, for all three quantities, Eq. (3). For $T \gtrsim 0.7 T_{c}$, the effective asymptotic growth exponent decreases, and it becomes smaller going from $L(t)$ to $\Delta E(t)$ to $R(t)$. This is indicated in the composite plot in Fig. 4. A similar decrease of the effective growth exponent for $T$ approaching $T_{c}$ was encountered in the study by Sadiq and Binder. ${ }^{8}$ The large error bars in Fig. 4 at high temperatures reflect the

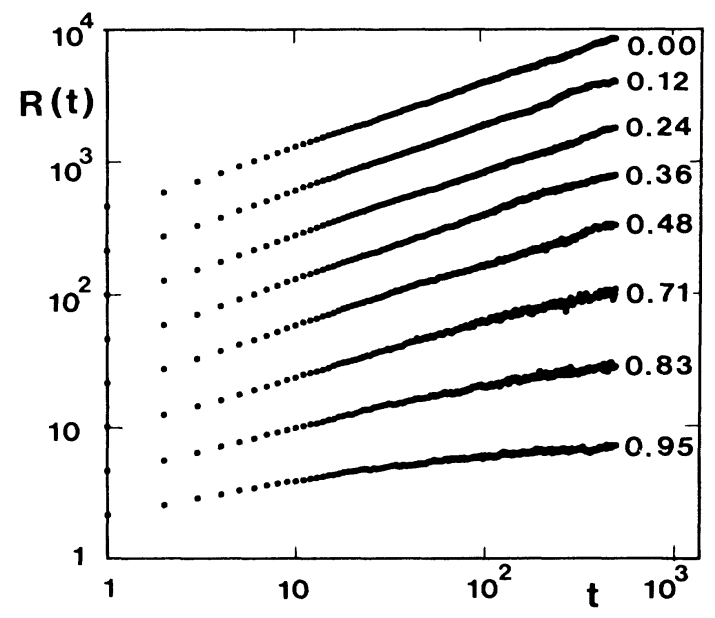

FIG. 3. Log-log plot of the linear domain size $R(t)$ (in arbitrary units) vs time for selected quench temperatures $T_{f}$ in the case of $\alpha=1$. The data are obtained from Monte Carlo calculations on lattices with $100^{2}$ spins. The curves are labeled by the value of the reduced temperature $T_{f} / T_{c}$.

differences between the asymptotic effective growth exponents of the different quantities used to monitor the growth processes. Such differences arise as the temperature is raised toward the transition region due to influence of critical fluctuations. As shown by Sadiq and Binder, ${ }^{8}$ this may be accounted for in the critical region by the introduction of the dynamic exponent $z$ via the concept of extended dynamic scaling. ${ }^{29}$ Our data in this temperature region are not accurate enough to sustain a quantitative analysis along these lines.

The freezing-in phenomena found for quenches to zero temperature for $\alpha<1$ are associated with metastable

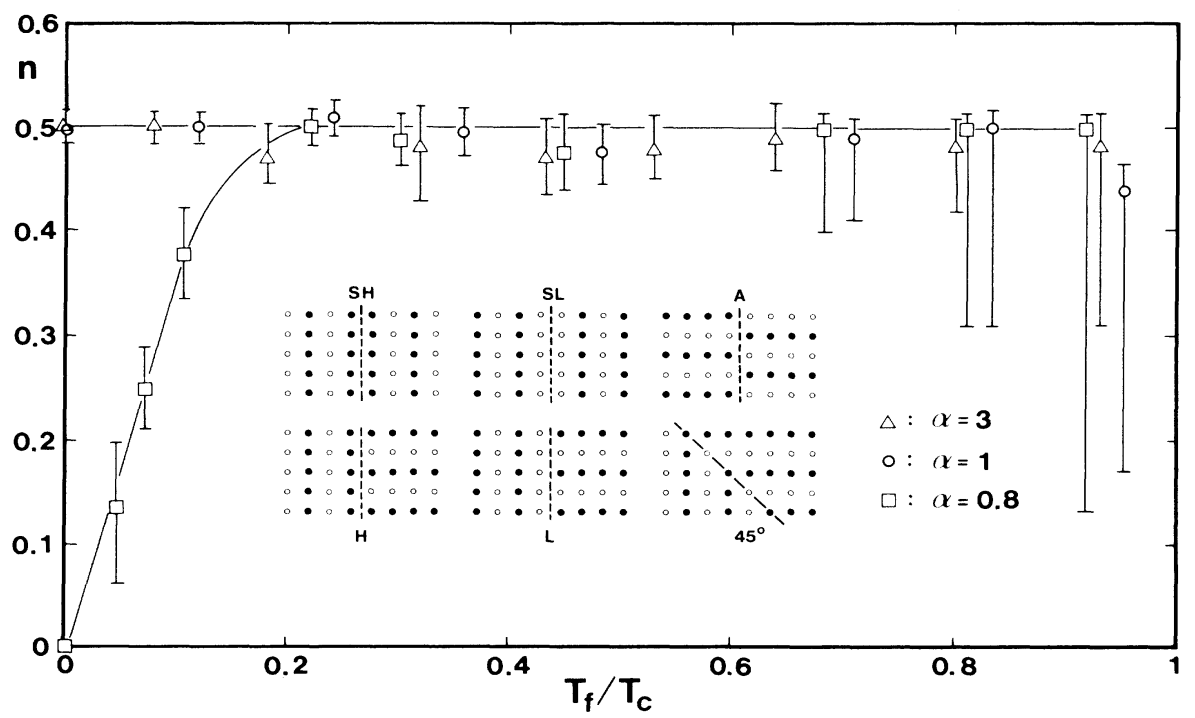

FIG. 4. Kinetic growth exponent $n$ as a function of reduced quench temperature $T_{f} / T_{c}$. Results are shown for three different values of $\alpha . \circ, \alpha=1 ; \Delta, \alpha=3$; and $\square, \alpha=0.8$. The large error bars for $T \gtrsim 0.7 T_{c}$ reflect the differences between the asymptotic effective growth exponents of the different quantities. The inset shows the six different types of domain walls: superheavy (SH), superlight (SL), antiphase (A), heavy (H), light (L), and $45^{\circ}$ walls. 
glassy polycrystalline domain structures in which the domain walls are pinned. Unpinning and resumption of growth result when thermal fluctuations are introduced at finite temperatures. This is clearly demonstrated by the data for $\alpha=0.8$, also included in Fig. 4. The effective exponent rises from a very low value at $T_{f}=0$ to the expected value of $n=0.5$ at $T_{f}=0.25 T_{c}$. For temperatures above this value, the kinetic exponent values are the same as those obtained for $\alpha=1$. A similar behavior is found for lower values of $\alpha$. However, the rise of $n$ towards $n=\frac{1}{2}$ is slower the smaller the value of $\alpha$. For comparison, Fig. 4 also includes data for $\alpha=3$ showing that the temperature dependence of $n$ for $\alpha \geq 1$ is the same as that for $\alpha=1$. In the language of renormalization-group analysis of domain-growth kinetics, ${ }^{9,27}$ these results could be rephrased: For $\alpha \geq 1$, the dynamics for all $T<T_{c}$ is governed by an equilibrium fixed point characterized by $n=\frac{1}{2}$. For $\alpha<1$, the zero-temperature growth is controlled by a freezing fixed point; whereas at any finite temperature the freezing fixed point becomes unstable and the basin of attraction of the equilibrium fixed point includes all initial unstable states; when the temperature is close to zero, the early-time kinetics is strongly influenced by a crossover from one fixed point to the other.

In conclusion, we have presented computer-simulation results for the domain-growth kinetics of the $(2 \times 1)$ ordering process as it takes place in a two-dimensional square Ising model with antiferromagnetic next-nearestand nearest-neighbor interactions with a strength ratio of $\alpha$. We have shown that the growth exponent assumes the classical value of $n=\frac{1}{2}$ independent of temperature for $\alpha \geq 1$. For $\alpha<1$, there are dramatic freezing-in effects at zero temperature which, however, may be released as the temperature is increased. This latter observation may be of some relevance to experimental studies of the ordering dynamics in $\mathrm{O}$ overlayers chemisorbed on $\mathrm{W}(110)$. For such overlayers it is found ${ }^{18}$ that there is no ordering at low temperatures, but that order develops after uptemperature quenching to higher temperatures below $T_{c}$. This is in accordance with our results in Fig. 4, considering that the condition of $\alpha<1$ is likely to apply for $\mathrm{O}$ on W(110). ${ }^{30}$

This work was supported by the Danish Natural Science Research Council under Grant No. 5.21.99.72.
*To whom correspondence should be sent.

${ }^{1}$ For a review, see J. D. Gunton, M. San Miguel, and P. S. Sahni, in Phase Transitions and Critical Phenomena, edited by C. Domb and J. L. Lebowitz (Academic, New York, 1983), Vol. 8, p. 269.

${ }^{2}$ M. K. Phani, J. L. Lebowitz, M. H. Kalos, and O. Penrose, Phys. Rev. Lett. 45, 366 (1980).

${ }^{3}$ P. S. Sahni, G. Dee, J. D. Gunton, M. Phani, J. L. Lebowitz, and M. Kalos, Phys. Rev. B 24, 410 (1981).

${ }^{4}$ T. Ohta, D. Jasnow, and K. Kawasaki, Phys. Rev. Lett. 49, 1223 (1982).

${ }^{5}$ P. S. Sahni, G. S. Grest, and S. A. Safran, Phys. Rev. Lett. 50, 60 (1983).

${ }^{6}$ P. S. Sahni and J. D. Gunton, Phys. Rev. Lett. 47, 1754 (1981).

${ }^{7}$ K. Kaski, M. C. Yalabik, J. D. Gunton, and P. S. Sahni, Phys. Rev. B 28, 5263 (1983).

${ }^{8}$ A. Sadiq and K. Binder, Phys. Rev. Lett. 51, 674 (1983); J. Stat. Phys. 35, 517 (1984).

${ }^{9}$ G. F. Mazenko and O. T. Valls, Phys. Rev. B 30, 6732 (1984).

${ }^{10}$ G. S. Grest and D. J. Srolovitz, Phys. Rev. B 30, 5150 (1984).

${ }^{11}$ E. T. Gawlinski, M. Grant, J. D. Gunton, and K. Kaski, Phys. Rev. B 31, 281 (1985).

12 J. Viñals and J. D. Gunton, Surf. Sci. 157, 473 (1985).

${ }^{13}$ K. Kaski, T. Ala-Nissilä, and J. D. Gunton, Phys. Rev. B 31, 310 (1985)

${ }^{14}$ G. F. Mazenko, O. T. Valls, and F. C. Zhang, Phys. Rev. B 31, 4453 (1985).

${ }^{15}$ T. Ala-Nissilä, J. D. Gunton, and K. Kaski, Phys. Rev. B 33, 7583 (1986).

${ }^{16}$ E. T. Gawlinski, S. Kumar, M. Grant, J. D. Gunton, and K. Kaski, Phys. Rev. B 32, 1575 (1985).

${ }^{17}$ S. R. Anderson and G. F. Mazenko, Phys. Rev. B 33, 2007 (1986).

${ }^{18}$ P. K. Wu, J. H. Perepezko, J. T. McKinney, and M. G. Lagally, Phys. Rev. Lett. 51, 1577 (1983).

${ }^{19}$ C. Fan and F. Y. Wu, Phys. Rev. 179, 560 (1969); D. P. Lan- dau, J. Appl. Phys. 42, 1284 (1971); K. Binder and D. P. Landau, Phys. Rev. B 21, 1941 (1980).

${ }^{20} \mathrm{~K}$. Binder, in Monte Carlo Methods in Statistical Physics, edited by K. Binder (Springer-Verlag, Heidelberg, 1986), p. 1.

${ }^{21}$ For a review, see O. G. Mouritsen, Computer Studies of Phase Transitions and Critical Phenomena (Springer, Heidelberg, 1984).

${ }^{22}$ The accordance between the kinetic exponent value obtained here using the Metropolis criterion and the value obtained in Ref. 8 using conventional Glauber dynamics (heat-bath method) lends evidence to the concept of universality in domain-growth kinetics, i.e., details of the excitation mechanism are irrelevant. In contrast, the amplitudes of the growth laws are dependent on these details.

${ }^{23}$ I. M. Lifshitz, Zh. Eksp. Teor. Fiz. 42, 1354 (1962) [Sov. Phys. JETP 15, 939 (1962)]; S. A. Allen and J. W. Cahn, Acta Metall. 27, 1085 (1979).

${ }^{24}$ P. S. Sahni, D. J. Srolovitz, G. S. Grest, M. P. Anderson, and S. A. Safran, Phys. Rev. B 28, 2705 (1983).

${ }^{25}$ O. G. Mouritsen, Phys. Rev. B 32, 1632 (1985).

${ }^{26}$ O. G. Mouritsen, Phys. Rev. B 31, 2613 (1985); Phys. Rev. Lett. 56, 850 (1986).

${ }^{27}$ J. Viñals and J. D. Gunton, Phys. Rev. B 33, 7795 (1986).

${ }^{28}$ The sensitivity of our computer simulation results to the quality of the random number generator used to simulate the stochastic motion of the domain walls has been assessed by employing different pseudorandom number generators [cf. also A. Milchev, K. Binder, and D. W. Hermann, Z. Phys. B 63, 521 (1986)]. It turns out that the quality of the random numbers is of little importance at low temperatures, but of decisive importance as the temperature is raised above $T \simeq 0.7 T_{c}$.

${ }^{29}$ P. C. Hohenberg and B. I. Halperin, Rev. Mod. Phys. 49, 435 (1977).

${ }^{30}$ W. Y. Ching, D. L. Huber, M. G. Lagally, and G.-C. Wang, Surf. Sci. 77, 550 (1978); E. D. Williams, S. L. Cunningham, and W. H. Weinberg, J. Chem. Phys. 68, 4688 (1978). 